\title{
Agent Behavior-Based Simulation Study on Mass Collaborative Product Development Process
}

\author{
Shuo Zhang, ${ }^{1}$ Yingzi Li, ${ }^{2}$ and Xiaodong Zhang ${ }^{2}$ \\ ${ }^{1}$ School of Economics and Management, North China Electric Power University, Beijing 102206, China \\ ${ }^{2}$ Dongling School of Economics and Management, University of Science and Technology Beijing, Beijing 100083, China \\ Correspondence should be addressed to Yingzi Li; liyz@ustb.edu.cn
}

Received 23 January 2015; Revised 1 March 2015; Accepted 2 March 2015

Academic Editor: Chin-Chia Wu

Copyright (c) 2015 Shuo Zhang et al. This is an open access article distributed under the Creative Commons Attribution License, which permits unrestricted use, distribution, and reproduction in any medium, provided the original work is properly cited.

\begin{abstract}
Mass collaborative product development (MCPD) benefits people by high innovation products with lower cost and shorter lead time due to quick development of group innovation, Internet-based customization, and prototype manufacturing. Simulation is an effective way to study the evolution process and therefore to guarantee the success of MCPD. In this paper, an agent behavior-based simulation approach of MCPD is developed, which models the MCPD process as the interactive process of design agents and the environment objects based on Complex Adaptive System (CAS) theory. Next, the structure model of design agent is proposed, and the modification and collaboration behaviors are described. Third, the agent behavior-based simulation flow of MCPD is designed. At last, simulation experiments are carried out based on an engineering case of mobile phone design. The experiment results show the following: (1) the community scale has significant influence on MCPD process; (2) the simulation process can explicitly represent the modification and collaboration behaviors of design agents; (3) the community evolution process can be observed and analyzed dynamically based on simulation data.
\end{abstract}

\section{Introduction}

Mass collaborative product development (MCPD), also called open source design, is an emerging design mode in recent years. In MCPD, a mass number of volunteer designers with complementary knowledge develop product autonomously and complete product creation, design, test, and even popularization together by means of open network platform [1]. MCPD has developed rapidly with advantage of high innovation, low cost, and high customer satisfaction. Thus, MCPD has become an important complementary mode of traditional collaborative product development (CPD) gradually. Different from the top-down organization mode of traditional CPD, MCPD is in bottom-up self-organized structure. Moreover, MCPD can make full use of the mass emergence of design originality as well as sharing of technology, resource, and knowledge between designers.

At present, Open Source Software (OSS) is the most successful application of MCPD, and some open source communities (OSC) that coexist with OSS are in effective operation like Linux, Apache, Mozilla, and so on. In recent years, MCPD has been applied in industrial product design. In this mode, the new product originality or CAD model is released publicly by individuals or enterprises, and a mass of OSC members will complete it collaboratively based on conceptual product. Many new ideas and products emerge constantly in some famous innovation OSC such as Open Source Hardware, Open Source Car, Prosthetics Project, and Lego Mindstorms. These cases show that MCPD is influencing product development mode deeply. Based on these successful cases, many scholars have made fruitful research on human factors of MCPD process in recent years. In this domain, Li et al. [2] proposed and tested a model that could be used to assess the relationship between an OSS project leader's leadership style and a developer's motivation; Santos et al. [3] developed a theoretical model to explore the contextual and causal factors of project attractiveness in inducing activities and tested it with data from Sourceforge; Gallego et al. [4] developed a technological acceptance model on behalf of the users towards a solution based on OSS; Midha and Palvia [5] developed a comprehensive research model including both extrinsic and intrinsic attributes that led to OSS success; Wang [6] used a large data set of Free Open Source Software (FOSS) projects obtained from http://SourceForge.net/ to investigate survival 
factors at various stages of a FOSS project's lifecycle. Overall, the above research mainly focuses on study of participation motivation and factors of MCPD in qualitative aspect, which lays preliminary foundation for further research on designer's microcosmic interactive behaviors as well as interactions in MCPD process. Meanwhile, many OSC suffer failure, as study on operation mechanism and evolution principles of MCPD is still in exploratory stage and collective intelligence has not been used efficiently.

In this paper, an agent behavior-based modeling and simulation method is proposed to study operation mechanism and evolution principles of MCPD based on theory of Complex Adaptive System (CAS). In MCPD process, designers are the most active and flexible elements, in which they build complex relationships by collaborative behaviors and promote product evolution by interactions with environment that mainly consists of product project and design resources. As designer behaviors directly influence evolution of MCPD process, agent behavior-based simulation becomes an effective way to improve process efficiency by analyzing interactive behaviors based on quantitative simulation results.

The rest of the paper is organized as follows. In Section 2 related work on modeling and simulation study of open innovation and MCPD process are discussed. In Section 3 agent behavior-based simulation model of MCPD process is proposed. In Section 4 the structure model of design agent is described in detail, and behaviors of modification and collaboration are illustrated. In Section 5 simulation program is developed in combination with the complex process of MCPD and dynamic interactions between agents. In Section 6, simulation experiments based on an engineering case of mobile phone design are designed, and related simulations are carried out to analyze community factor, designer's dynamic characteristics, and community evolution in MCPD process. In Section 7, the highlights and future work are represented.

\section{Related Work}

2.1. Open Innovation. MCPD is a typical kind of open innovation, and current studies focus on formation and evolution mechanism of cluster innovation network. In these studies, Social Network Analysis (SNA) is the most common method, which describes interactions between individuals as nodes (representing individuals) and ties (representing relationships between the individuals) based on network theory. For example, van der Valk et al. [7] developed a framework for evaluation and analysis of innovation networks based on SNA; Toral et al. [8] carried out macro- and microstructural analysis to study the behavior of virtual communities for OSS projects applying SNA; König et al. [9] built a model for network evolution of firms exchanging knowledge based on marginal revenues and costs and studied the characteristics of equilibrium networks applying SNA. In addition, many scholars pay close attention to efficiency of open innovation network and enthusiasm of members. For example, Ye and Kankanhalli [10] investigated the potential factors that could promote solvers' and seekers' participation in open networks; Martínez-Torres [11] proposed the use of evolutionary computation techniques for the identification of innovators with the ability of generating attractive and applicable ideas for the organization; Wi et al. [12] presented a virtual team formation model as a quantitative and systematical method of selecting virtual team members for open innovation and verified its efficiency; Bianchi et al. [13] developed a model describing the adoption of Open Innovation by biopharmaceutical companies based on two rounds of interviews with industry experts and analyzed knowledge interaction in different organizational modes of biopharmaceutical firms; Choi et al. [14] developed a simple computational model of social networks and proposed computational approach incorporating small-world graphs to find that diffusion of innovation was more likely to fail in a random network than in a highly clustered network of consumers. Raasch et al. [15] explored the evolution of open source research on interdisciplinary coauthorship and cocitation and publication patterns by use of objective bibliometric data and other secondary data.

The aforementioned work provides a lot of valuable research on members' relationships and evolution models. In addition, although the referred collaborative innovation behaviors that derive from knowledge complementarity and potentials of innovation clusters represent system evolution mechanism, they are mainly about enterprises, not individual designers. Meanwhile, the above researches are focused on evolution of knowledge and innovation process, which overlooks evolution process of product.

2.2. Modeling and Simulation. With rapid development of open innovation, scholars apply methods of modeling and simulation to study operation mechanism of various open innovation modes with quantitative results. For example, Scacchi [16] introduced and examined some of the issues when seeking to discover and computationally model the software engineering processes; Le and Panchal [17] presented an approach for generating surrogate bipartite networks with varying sizes based on degree distributions of given bipartite networks and illustrated it using a bipartite network from an open source software development (OSSD) repository; Sack et al. [18, 19] described a methodological framework and developed a model to analyze the collaborative design process of OSS. In addition, Haythornthwaite [20] presented two models of collaborative behaviors pointing to structures and motivators that operated on different aspects of peer productions; Li and Wang [21] developed a system dynamics model of general peer production by analyzing dynamic mechanism of peer production with system dynamics theory; Yang et al. [22] extended the complex network theory and modeling on the research of general viral marketing and developed a specific spreading scheme and an approach based on a real complex network.

The above researches are focused on process modeling but members' behaviors are rarely studied. To study dynamic evolution of system process, agent-based models (ABM) methodology is approached. As System Complexity derives from the flexibility of agents, the evolution process can be described by modeling agents' behaviors. In recent years, ABM has been used to deal with problems such as power 
distribution, manufacturing, finance, and natural resource management [23-30]. The primary advantage of an agentbased simulation is that it allows the study of emergent behavior of complex systems in a bottom-up fashion [31]. $\mathrm{ABM}$ has been shown to deal with problems of complexity and openness, with dynamical and unknown environments changing over time [32]. In product development domain, Levitt et al. [33, 34] proposed Virtual Design Team (VDT) with $\mathrm{ABM}$ so as to carry out study on modeling and simulation of product design process. Martínez-Miranda and Pavón [35] proposed an agent-based model where a virtual team can be configured using the characteristics of the real candidates to form the team, and given a set of tasks, the model generates the possible performance of the team members. Garcia [36] discussed a possible use of agent-based methodology in innovation and new product development research. Wang et al. [37] built a new framework for collaborative design adopting an agent-based approach based on the analysis of the dynamic nature of collaborative design process. However, compared to traditional product development, both process and members' behaviors are more complex in MCPD. Based on the characteristics of MCPD, Panchal [38] has done valuable researches by agent simulation. The referred models and simulation generally represent autonomy and complexity of MCPD in macroscopic perspective. But the description to designers' microscopic behaviors is simplified, and interactions of behaviors are not described.

\section{Agent Behavior-Based Simulation Model of MCPD Process}

MCPD is a typical knowledge collaboration process, in which masses of designers autonomously select to participate in product development of OSC in consideration of their personal attributes like knowledge level and technical ability and perform interactive collaboration with other designers. Therefore, designers are defined as design agents in the agent behavior-based simulation model, which packages them into three types, management agent, technical core agent, and common development agent, based on their different behavior roles in MCPD. Management agent mainly performs behaviors like community maintenance, conflict negotiation, project subdivision, module task screening and releasing, and so on; technical core agent contributes to MCPD with behaviors of technical review and key module task development and collaboration; common development agent plays his role by product feedback and module task development and collaboration. In MCPD process, the three kinds of agents collaborate interactively, promoting evolution of product and OSC.

The agent behavior-based simulation model of MCPD process is built as shown in Figure 1, which is composed of two parts: design agents and environment objects. During MCPD process, design agents perceive environment objects' information (e.g., project, module task, and resource) continuously and perform corresponding behaviors in combination with structure model of design agent to update the information, thus promoting evolution of MCPD.

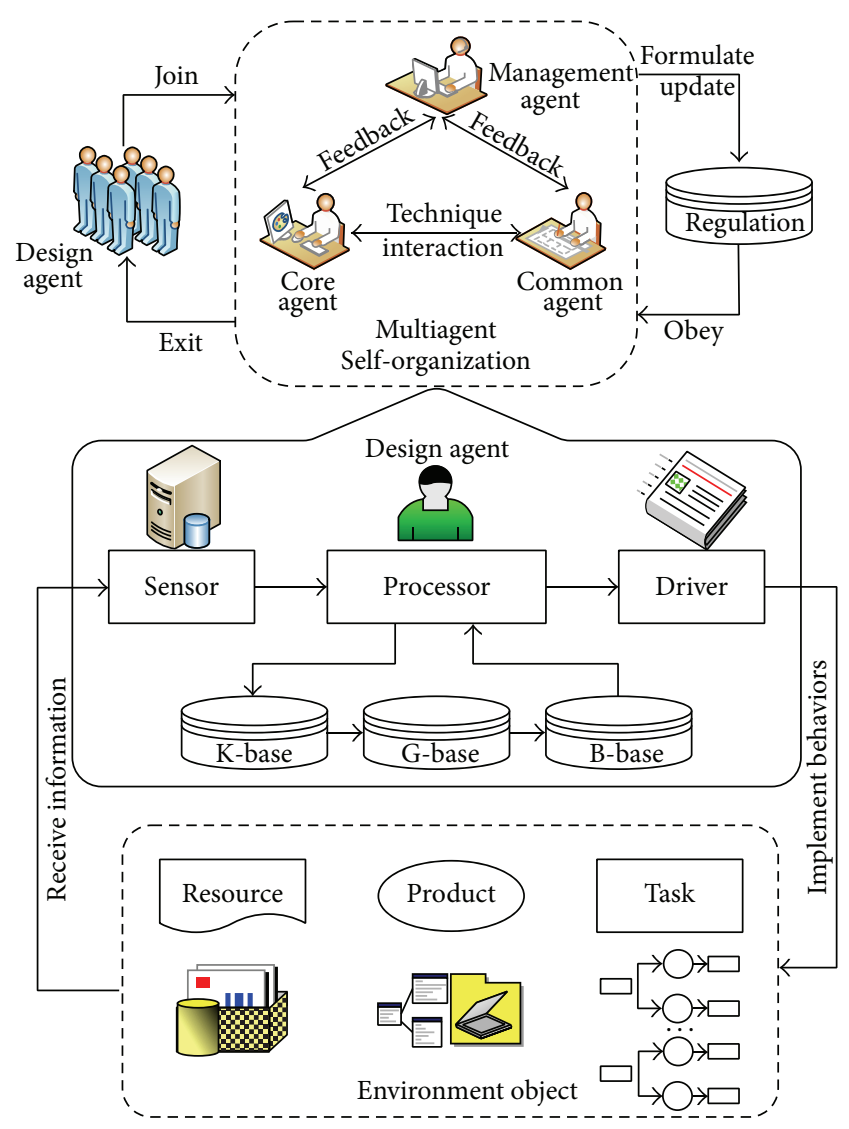

FIGURE 1: Agent behavior-based simulation model of MCPD process.

In Figure 1, environment objects are composed of product, module task, and resource. In this part, product object is mainly constituted by mathematical models and related documents, which are shared by OSC members; task object consists of a series of module tasks, which are packaged according to requirements of product project and completed autonomously by design agents during MCPD process; resource object mainly refers to various resources used or shared by design agents, such as design tools, software, experimental equipment, and rapid prototyping equipment.

According to above simulation model shown in Figure 1, design agent receives information from environment objects by Sensor and performs corresponding behaviors to update information of environment objects by Driver. Between the two phases, design agent selects correct behaviors by Processor according to information of Sensor. In Processor phase, design agent makes decision to select behaviors by structure model of design agent which is described in Section 4. In this way, MCPD evolution including product evolution and community evolution is carried out by design agents' complex behaviors.

\section{Structure Model of Design Agent}

In Processor phase of agent behavior-based simulation model of MCPD process, behavior decision is made according to structure model of design agent as shown in Figure 2. 


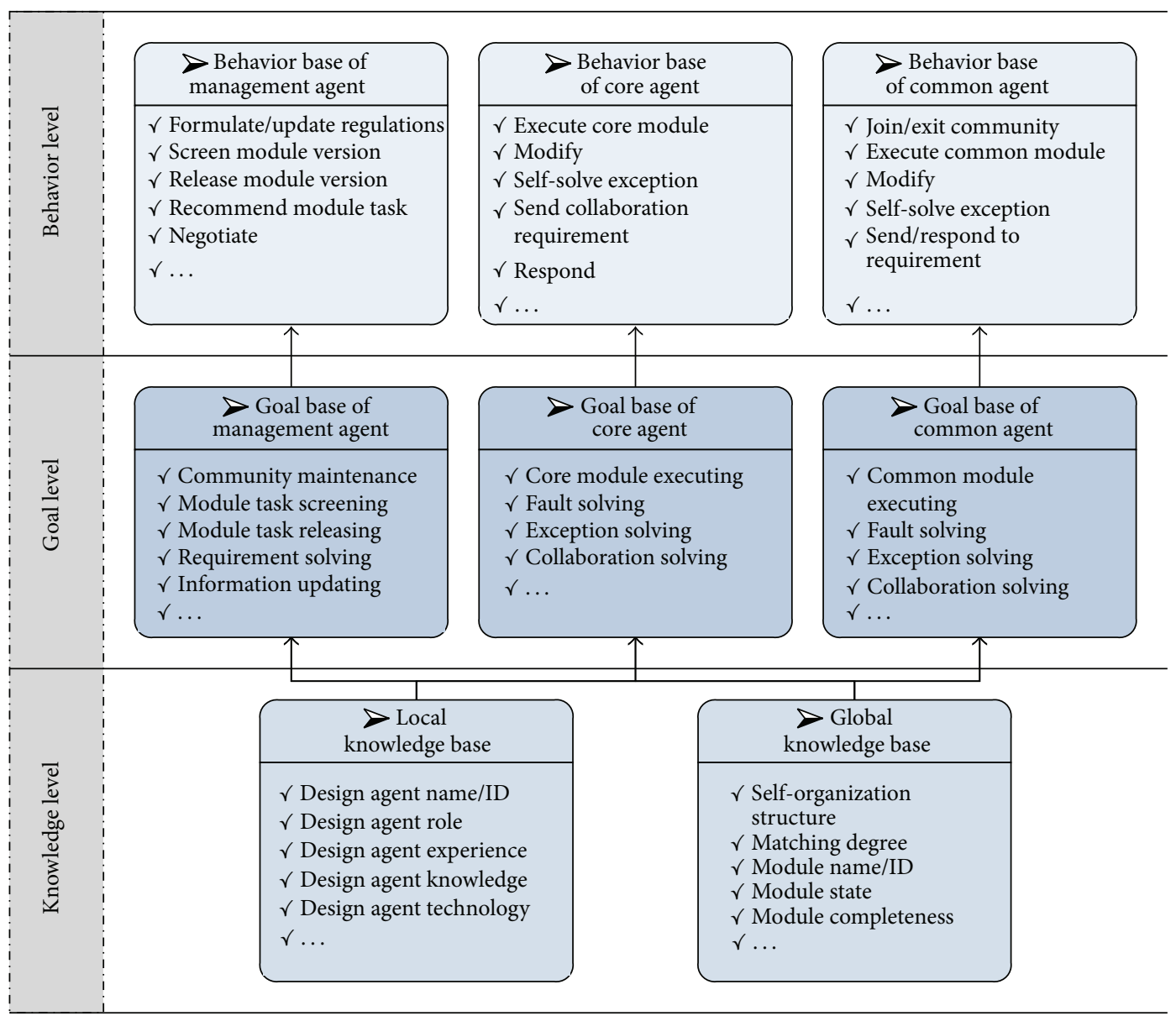

FIGURE 2: Structure model of design agent.

In structure model, there are three levels, knowledge level, goal level, and behavior level, by which design agent invokes corresponding bases to make decisions. In Knowledge level, there are two knowledge bases, the local one storing design agent's basic attributes, such as experience value, knowledge vector, and technology vector, and the global one storing attributes of environment object and other agents that design agent can get directly. In Goal level, there are three bases corresponding to the three kinds of design agents, and the goal is extracted in consideration of both knowledge level and information received. In Behavior level, there are also three bases corresponding to the three kinds of design agents, which contain agent's complex behaviors during MCPD process. Based on the goal extracted in Goal level, design agent selects correct behavior in this level corresponding to information received. After decision of behavior selection, design agent performs corresponding behavior to promote product evolution.

In MCPD, agents' various behaviors directly represent their autonomy and complexity. Among these behaviors [39], modification behavior and collaboration behavior are the two most typical ones to represent MCPD's autonomy and complexity. The description to the two behaviors is as follows.

(1) Modification Behavior. It refers to rework process for fault while design agent performs module task as shown in

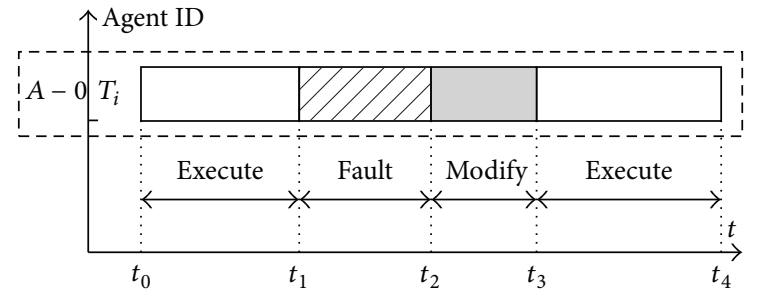

FIGURE 3: Modification behavior flow.

Figure 3. Design agent $A-0$ (0 denotes Agent ID) starts executing module task $T_{i}(i=0,1,2, \ldots)$ at $t_{0}$ and completes it at $t_{4}$. During the process, he finds that some faults occur while executing it in duration $\left[t_{1}, t_{2}\right]$. As a result, he has to rework to modify the fault segment in the duration $\left[t_{2}, t_{3}\right]$, which is shorter than the fault segment for learning effect. After modification, $A-0$ continues executing $T_{i}$ regularly and finishes it at $t_{4}$ finally.

Because of learning effect, time and cost to complete the modification segment will be gradually reduced. The learning process usually presents as a negative exponential curve. In order to quantitatively describe the learning effect, there are lots of models for different needs, such as power index model, Stanford B model, exponential model, S curve model, 


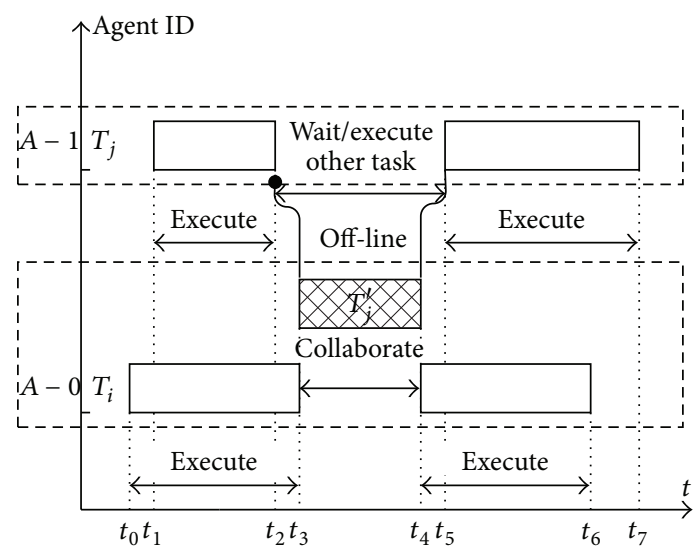

FIGURE 4: Off-line collaboration behavior flow.

and time constant model [40]. In this paper, time constant model is selected to describe learning effect [26], which can intuitively display the learning speed. The model for learning effect is as shown in formula (1). Consider

$$
d_{k}=d_{1}-d_{F}\left[1-\exp \left(\frac{-k}{k_{0}}\right)\right] .
$$

$d_{1}$ is the initial time to complete modification segment; $d_{F}$ is the time to complete modification segment after learning something; $d_{k}$ is the time to complete modification segment after $k$ times execution of a task; $k_{0}$ is the learning cycle, which points out the task execution times to improve work performance by the design agent and reflects the agent's learning speed and ability.

(2) Collaboration Behavior. While executing module task, design agent may encounter some difficulties for knowledge, technology, and so on, which he cannot figure out independently. As a result, he has to seek help for collaboration to resolve the difficulties. There are two ways of collaboration, which are off-line collaboration behavior and on-line collaboration behavior, respectively.

(a) Off-Line Collaboration Behavior. As shown in Figure 4, $A-1$ encounters difficulties at $t_{2}$ while executing $T_{j}$ and has to send collaboration requirement to figure them out. In comprehensive consideration of the current problem's difficulty level (high), possible duration (not short), and urgency (not high), he packages the collaboration requirement into off-line collaboration task $T_{j}^{\prime}$. After $A-0$ receives $T_{j}^{\prime}$, he responds to $A-1$ at $t_{3}\left(t_{3} \geq t_{2}\right)$, suspends the current task $T_{i}$, and turns to complete $T_{j}^{\prime}$ in duration $\left[t_{3}, t_{4}\right]$ independently; at the same time, $A-1$ just waits for collaboration or turns to execute other module tasks. After $T_{j}^{\prime}$ is completed by $A-0, A-1$ turns to continue $T_{j}$ at $t_{5}$.

(b) On-Line Collaboration Behavior. As shown in Figure 5, $A-1$ encounters difficulties at $t_{2}$ while executing $T_{j}$ and has to send collaboration requirement to figure them out. In comprehensive consideration of the current problem's difficulty level (not high), possible duration (short), and urgency

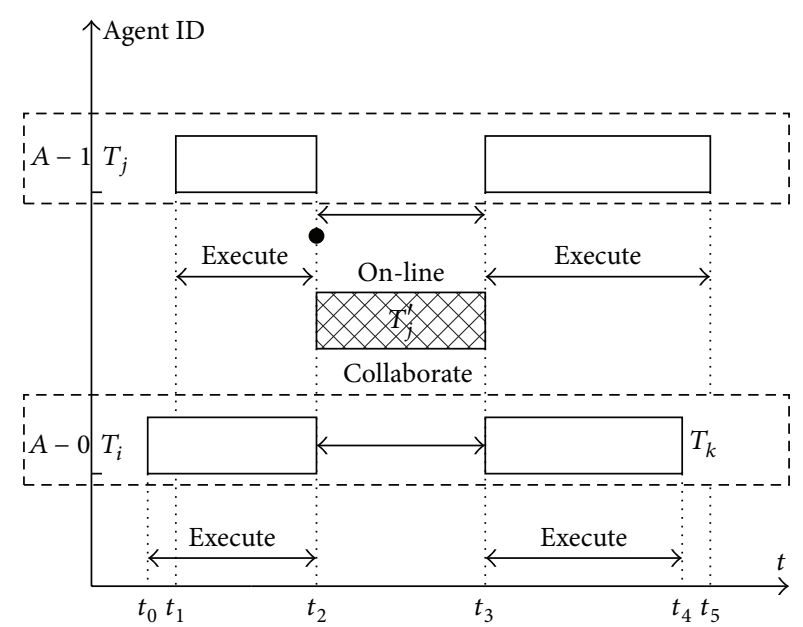

FIGURE 5: On-line collaboration behavior flow.

(high), he packages the collaboration requirement into online collaboration task $T_{j}^{\prime}$. After that, design agent sends $T_{j}^{\prime}$ to $A-0$ who is free (not executing task or just finishing it) for on-line collaboration. While $A-0$ receives $T_{j}^{\prime}$, he responds to $A-1$ at $t_{2}$, and then $A-0$ and $A-1$ resolve $T_{j}^{\prime}$ together in duration $\left[t_{2}, t_{3}\right]$. After on-line collaboration is completed, $A-1$ continues to perform $T_{j}$ while $A-0$ turns to other task $T_{k}$.

\section{Simulation Program Design}

Based on aforementioned models, simulation program for MCPD is developed with Visual $\mathrm{C}++6.0$, which packages design agents and environment objects, respectively, preliminarily realizing simulation of MCPD process.

The simulation flow of MCPD program is shown as follows in Figure 6, in which simulation process is timed by simulation step $(s p)$ and runs with continuous rounds until all module tasks are completed.

(1) Starting time of simulation is initialized to 0. In this stage, program operator sets initial parameters of product modules, design agents, and OSC.

(2) Management agent updates information of products, resources, tasks, design agents, and OSC.

(3) Design agent (technical core agent and common development agent) selects suitable tasks from OSC according to his preference, knowledge level, and technical level and adds them into task box.

(4) In consideration of task attributes, design agent (technical core agent and common development agent) chooses one optional task to execute from task box at current $s p$.

(5) Design agent executes the chosen task. After that, program returns to stage (3), continuing traversal of design agents (technical core agent and common development agent). 


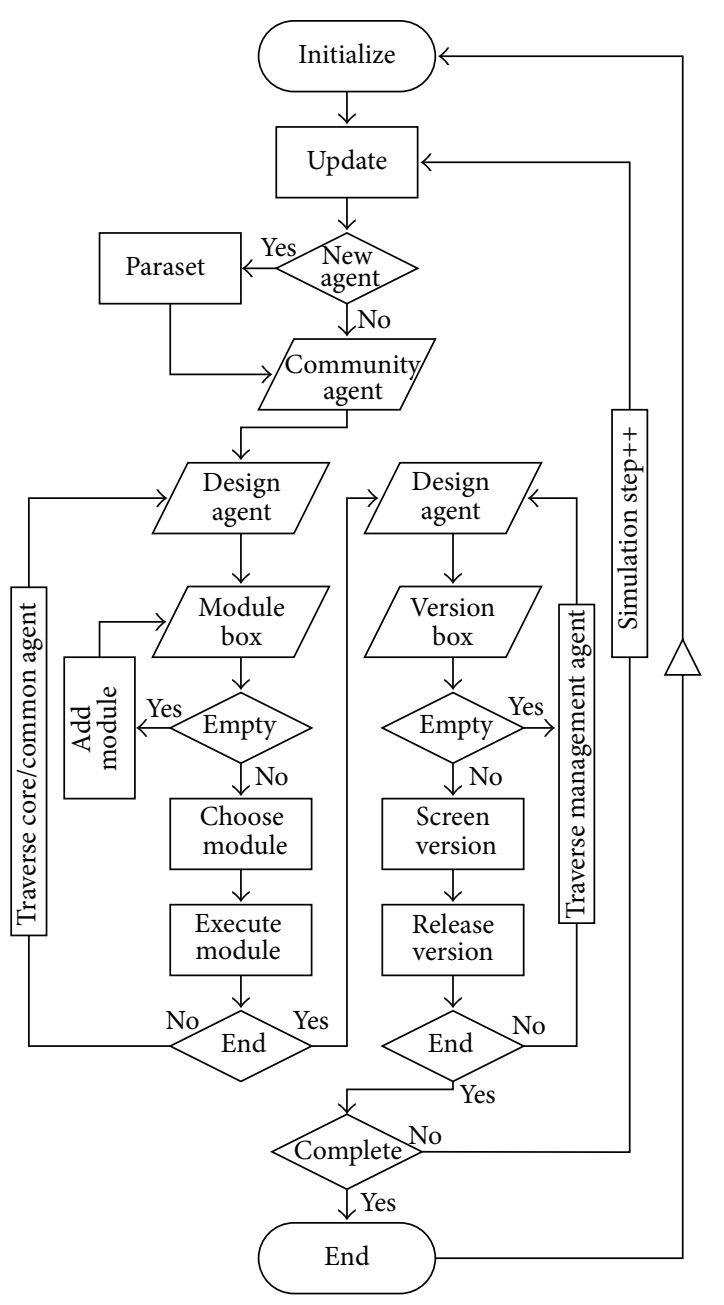

FIgURE 6: Simulation flow of MCPD process.

(6) Meanwhile, management agent performs management of module task version. In this stage, management agent firstly checks version box of completed modules, which may have multiple versions.

(7) In combination with module version's attributes like function, technology, and compatibility, management agent eliminates unqualified versions and retains qualified ones.

(8) Management agent releases the qualified versions to OSC to trigger subsequent module tasks.

(9) Program judges if all module tasks are completed. If so, project is completed, and this round of MCPD simulation ends.

\section{Case Study}

6.1. Simulation Experiment Design. To validate the agent behavior-based simulation model, a case study is modeled and simulated based on the engineering background of mobile phone design. A mobile phone usually consists of upper case, lower case, main board, speaker, keypad, antenna,

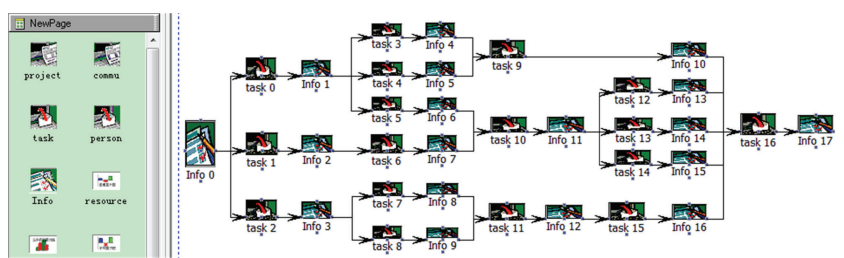

Figure 7: Product development flow.

TABLE 1: Initial parameter setting.

\begin{tabular}{lc}
\hline Parameter & Value \\
\hline Scenarios & S1, S2, and S3 \\
Initial scale of OSC & S1: 100; S2: 200; S3: 300 \\
Module task number & 17 \\
Task modification rate & $T_{i}: 0.2(i=0,1,2, \ldots, 16)$ \\
Task collaboration rate & $T_{i}: 0.15(i=0,1,2, \ldots, 16)$ \\
Simulation times & $300(100$ times each scenario $)$ \\
\hline
\end{tabular}

battery, Wi-Fi, camera, and so on. In this project, the design process is significantly dynamic and collaborative, and the project is decomposed into corresponding module tasks. To realize open source design, the project can be released to OSC and designed by mass volunteer designers. In such process, product evolves with OSC evolution, and collaborations between designers occur frequently. Hence, simulation experiment is designed and carried out based on the mobile phone design project. The product development flow is designed as shown in Figure 7.

As shown in Figure 7, the project is decomposed into 17 module tasks that are related to each other by lines, in which Task denotes module task and Info denotes the medium of related tasks. For example, if task 1 is finished, Info 2 records the task's status and triggers task 6 to be designed; otherwise, task 6 cannot be selected by designers until task 1 is finished. In addition, $T_{i}(i<=16)$ denotes module task, while $T_{i}(i>$ 16) denotes collaboration task. In simulation, $T_{i}(i>=0)$ could be selected by multiple design agents autonomously.

As shown in Table 1, 3 contrast scenarios (S1, S2, and S3) are designed for different initial OSC scale, in which the scale is set as 100, 200, and 300, respectively. In addition, task modification rate and collaboration rate of each module task are set as 0.2 and 0.15 , respectively. After parameter setting, each scenario is simulated 100 times, and the mass simulation data of design agents, tasks, and OSC are stored in SQL Server for further analysis.

\subsection{Simulation Experiment Analysis}

(1) One-Way Analysis of Variance for Impact of OSC Scale. With product evolution of MCPD, OSC scale is also in dynamic variation, which impacts product evolution in turn. To evaluate OSC scale's impact on product evolution, statistical hypothesis test is applied to analyze the efficiency by oneway analysis of variance (ANOVA). The hypothesis is as follows. 
TABLE 2: Result of one-way ANOVA for different OSC scale.

(a) Test of homogeneity of variances

\begin{tabular}{lccr}
\hline \multicolumn{2}{c}{ Sample } & & \\
Levene statistic & df1 & df2 & Sig. \\
\hline 1.173 & 2 & 297 & .311 \\
\hline
\end{tabular}

(b) ANOVA

\begin{tabular}{lccccc}
\hline & & Sample & & \\
& Sum of squares & df & Mean square & $F$ & Sig. \\
\hline Between groups & 20810.887 & 2 & 10405.443 & 17.160 & .000 \\
Within groups & 180089.710 & 297 & 606.363 & & \\
\hline Total & 200900.597 & 299 & & & \\
\hline
\end{tabular}

(c) Multiple comparisons

\begin{tabular}{|c|c|c|c|c|c|c|}
\hline \multirow{3}{*}{ (I) Group } & \multirow{3}{*}{$(J)$ Group } & \multicolumn{3}{|c|}{$\begin{array}{c}\text { Sample } \\
\text { LSD }\end{array}$} & \multirow{2}{*}{\multicolumn{2}{|c|}{ 95\% confidence interval }} \\
\hline & & \multirow{2}{*}{ Mean difference $(I-J)$} & \multirow{2}{*}{ Std. error } & \multirow{2}{*}{ Sig. } & & \\
\hline & & & & & Lower bound & Upper bound \\
\hline \multirow{2}{*}{1} & 2 & $20.1600000^{*}$ & 3.4824206 & .000 & 13.306654 & 27.013346 \\
\hline & 3 & $12.7900000^{*}$ & 3.4824206 & .000 & 5.936654 & 19.643346 \\
\hline \multirow{2}{*}{2} & 1 & $-20.1600000^{*}$ & 3.4824206 & .000 & -27.013346 & -13.306654 \\
\hline & 3 & $-7.3700000^{*}$ & 3.4824206 & .035 & -14.223346 & -.516654 \\
\hline \multirow{2}{*}{3} & 1 & $-12.7900000^{*}$ & 3.4824206 & .000 & -19.643346 & -5.936654 \\
\hline & 2 & $7.3700000^{*}$ & 3.4824206 & .035 & .516654 & 14.223346 \\
\hline
\end{tabular}

${ }^{*}$ The mean difference is significant at the 0.05 level.

$H_{0}: \mu_{1}=\mu_{2}=\mu_{3}$ (initial scale of OSC has no significant impact on product evolution).

$H_{1}: \mu_{1} \neq \mu_{2} \neq \mu_{3}$ (initial scale of OSC has significant impact on product evolution).

According to simulation with former experiment settings, 3 data sets of S1, S2, and S3 (initial scale: 100, 200, and 300) are extracted, and each set contains product development time of 100 simulations. One-way ANOVA is carried out with software SPSS, as shown in Table 2.

One-way ANOVA result shows that the null hypothesis is rejected and that initial OSC scale has significant impact on product evolution of MCPD with confidence level at 0.95 . Furthermore, $\mu_{1}=394.28$ (mean time of product development of S1) is significantly longer than mean values of S2 and $\mathrm{S} 3 ; \mu_{2}=374.12$ (mean time of product development of S2) is significantly shorter than mean values of S1 and S3; $\mu_{3}=$ 381.49 (mean time of product development of S3) is between mean values of S1 and S2. Given the former product development flow, S2 provides the best scale of three scenarios to promote MCPD process and shorten development time.

(2) Simulation of Design Agent's Behaviors in MCPD Process. To study design agent's behaviors in MCPD process, a set of data storing the whole product development process of $A_{10}$ $(\mathrm{ID}=10)$ in one simulation is extracted. By data processing, the behavior flow of $A_{10}$ in MCPD process is described in Figure 8.
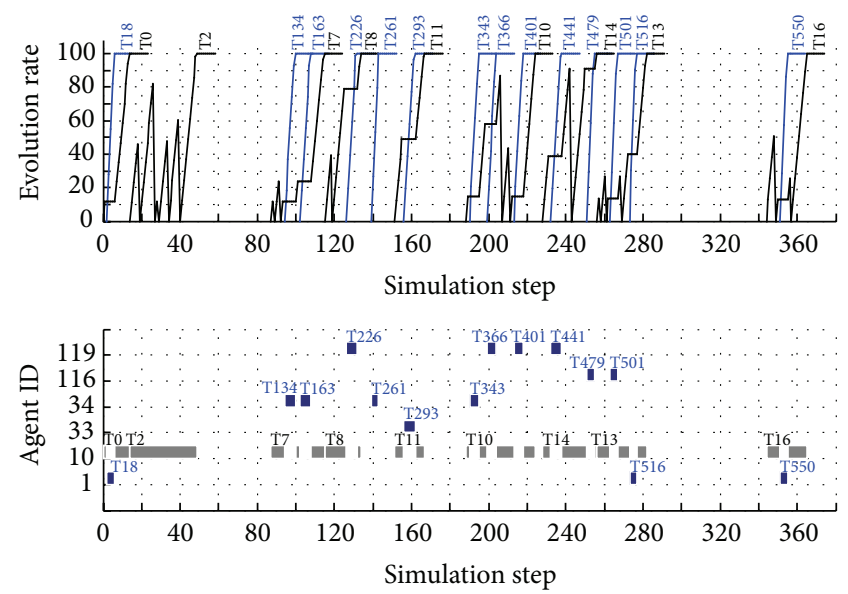

Figure 8: Behavior flow of $A_{10}$ in MCPD process.

As shown in Figure 8, vertical axes denote task evolution rate $(0-100 \%, 100 \%$ means the task is finished) and design agent ID, respectively, while horizontal axis denotes simulation time of MCPD. During the whole process, $A_{10}$ completes 9 module tasks $\left(T_{0}, T_{2}, T_{7}, T_{8}, T_{11}, T_{10}, T_{14}, T_{13}\right.$, and $\left.T_{16}\right)$ and 14 collaboration tasks $\left(T_{18}, T_{134}, T_{163}, T_{226}, T_{261}, T_{293}\right.$, $T_{343}, T_{366}, T_{401}, T_{441}, T_{479}, T_{501}, T_{516}$, and $\left.T_{550}\right)$. As shown in upper part of the figure, the black line charts represent evolution process of 9 module tasks executed by $A_{10}$, in which zigzag lines show the modification process for fault, straight 


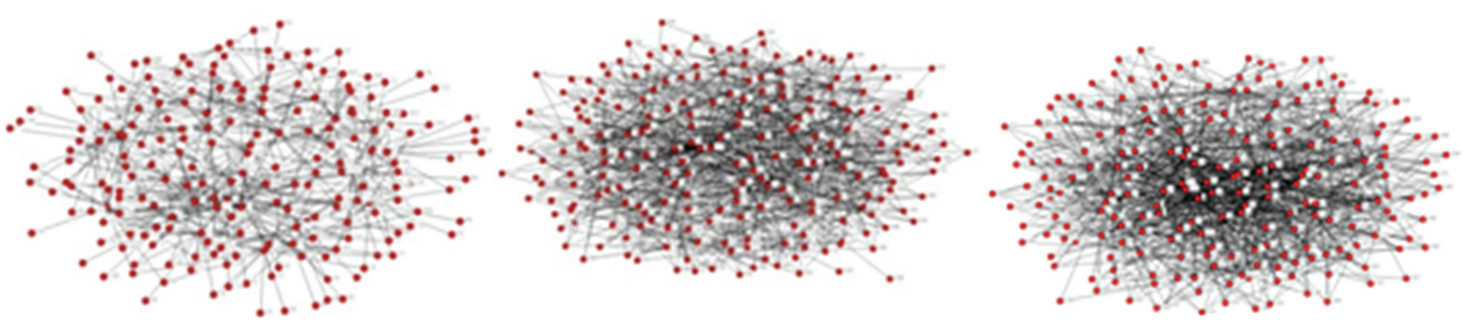

FIGURE 9: Social network diagrams at time 100, 200, and 300.

lines show that evolution of module tasks is paused for collaborations, and slope lines show tasks are executed by $A_{10}$ in regular way; the blue line charts represent 14 collaboration processes $A_{10}$ participates in, in which each collaboration task corresponding to the straight section of black line charts is sent by $A_{10}$ except that $T_{261}$ is sent by another agent and responded by $A_{10}$. As shown in lower part of the figure, gray sections represent execution process while $A_{10}$ executes the 9 module tasks, and blank sections indicate $A_{10}$ does not work on them for collaboration; blue sections represent 14 collaboration processes, in which $A_{10}$ collaborates with $A_{34}\left(T_{134}\right.$, $T_{163}, T_{261}$, and $\left.T_{343}\right)$ and $A_{119}\left(T_{226}, T_{366}, T_{401}\right.$, and $\left.T_{441}\right)$ each 4 times, $A_{1}\left(T_{18}, T_{516}\right.$, and $\left.T_{550}\right) 3$ times, $A_{116}\left(T_{479}\right.$ and $\left.T_{501}\right) 2$ times, and $A_{33}\left(T_{293}\right) 1$ time, respectively. In this simulation, design agent's regular execution behavior, modification behavior, and collaboration behavior in MCPD process are described in detail.

(3) Analysis of OSC Evolution. Along with evolution of module tasks in MCPD process, OSC also evolves for scale variation and relations between design agents. In OSC, relations between design agents are generated for collaborations. To study OSC evolution, a set of data storing relation matrices between design agents in one simulation is extracted. Social Network Analysis (SNA) is applied to analyze OSC evolution with software UCINET. Social network diagrams at simulation time 100, 200, and 300 generated by UCINET are shown in Figure 9.

As shown in Figure 9, the node denotes design agent of OSC, while the link denotes collaborative relationship between agents. Along with the evolution of MCPD process, relations among design agents become more and more frequent. As a result, the intensity of the social network diagrams becomes increasing.

In addition, simulation data of 5 time nodes (at time 50, $100,150,200$, and 300) are extracted and processed to calculate average distances, clustering coefficients, cliques, and densities, respectively, as shown in Figure 10.

In Figure 10, average distance between design agents is longest at step 150, and the reason for the phenomenon is that OSC scale is growing with product evolution, resulting in larger average distance. Meanwhile, collaboration between design agents becomes more frequent, especially when simulation time is over 150 , collaboration frequency continues to strengthen, and OSC scale is in slow growth, resulting in decrease of average distance. However, factors of clustering coefficient, clique, and density are all in growing trends with product evolution, which mainly results from continuous accumulation of collaboration relationships between design agents during MCPD process.

In one word, the simulation approach provides an effective way for MCPD study from an overall perspective, which helps study OSC evolution and its operation mechanism.

\section{Conclusions}

In this paper, the agent behavior-based simulation model of MCPD process and structure model of design agent are studied based on agent behaviors. The models describe designer's dynamic behavior characteristics, autonomy, initiative, and collaboration, and modification behavior and collaboration behavior are illustrated. Moreover, they represent uncertainty and complexity of MCPD process. The simulation proves that agent behavior-based simulation model of MCPD process and simulation program can describe MCPD process and its evolution from different perspectives like agent, product, and community.

Compared with existing literatures, the proposed approach is developed especially for MCPD process and has the following highlights: (1) community members' behaviors are considered from a microcosmic perspective. (2) Based on the quantitative simulation results, the MCPD process can be analyzed and evaluated efficiently.

Although the case study shows that the proposed approach is really a useful tool to describe and analyze the process on the basis of agent behaviors, the authors are conscious about the limitations of the approach. The behaviors of design agent should be enriched to keep the simulation process more realistic. During MCPD process, there may be conflicts such as resource conflict and partner selection conflict among design agents. For the problems, further study will be carried out. Moreover, more experiments should be explored to optimize the operation mechanisms of the MCPD process, such as encouragement, punishment, and collaboration mechanism.

\section{Conflict of Interests}

The authors declare that there is no conflict of interests regarding the publication of this paper. 

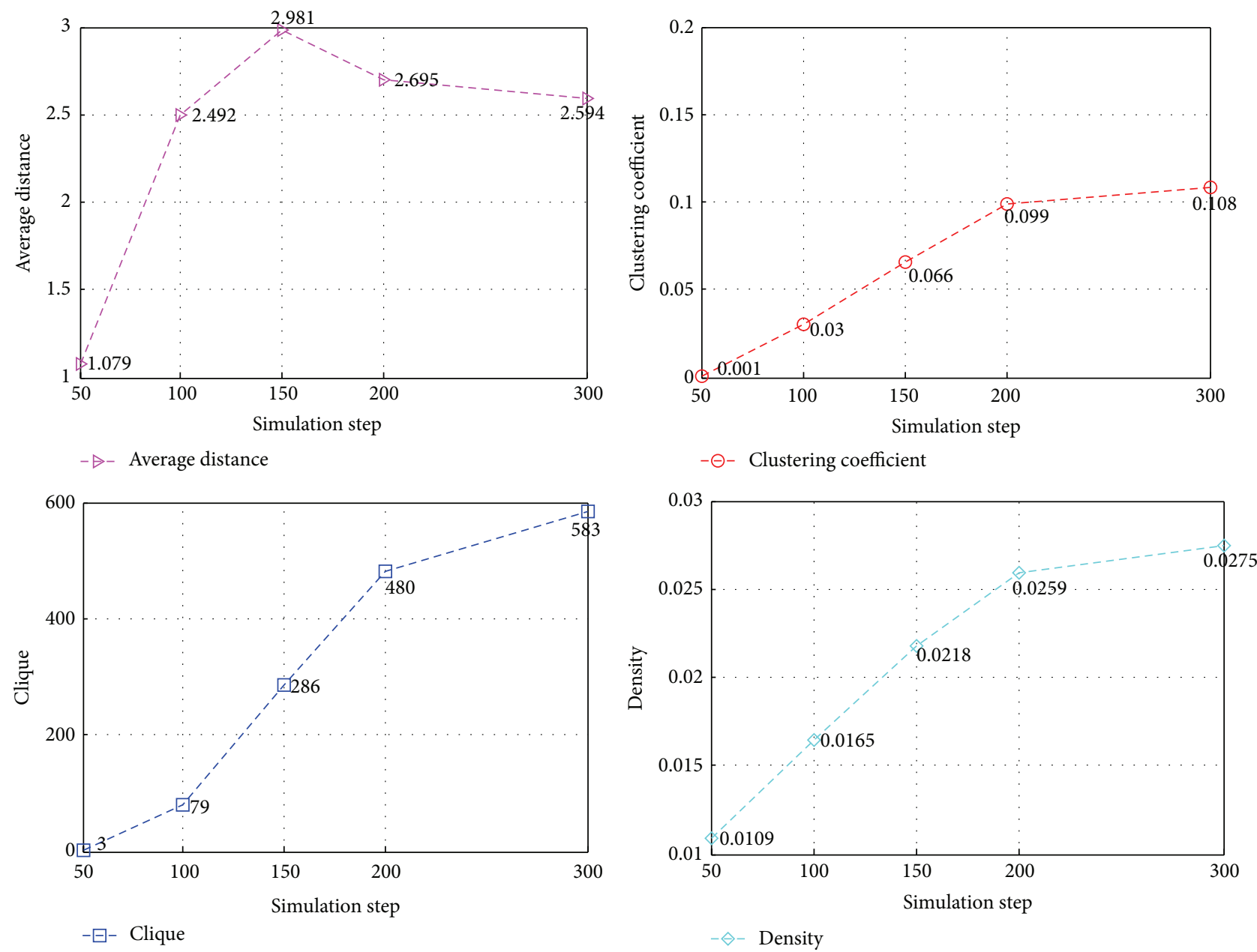

FIGURE 10: Line charts of SNA factors at 5 time nodes.

\section{Acknowledgments}

This work is based on research work supported by the National Natural Science Foundation of China under Grant no. 71171019; the Fundamental Research Funds for the Central Universities under Grant no. FRF-TP-14-055A2; and the Program for China Postdoctoral Science Foundation under Grant no. 2013T60064.

\section{References}

[1] D. Tapscott and A. D. Williams, Wikinomics: How Mass Collaboration Changes Everything, Portfolio, Penguin Group, 2006.

[2] Y. Li, C.-H. Tan, and H.-H. Teo, "Leadership characteristics and developers' motivation in open source software development," Information and Management, vol. 49, no. 5, pp. 257-267, 2012.

[3] C. Santos, G. Kuk, F. Kon, and J. Pearson, "The attraction of contributors in free and open source software projects," The Journal of Strategic Information Systems, vol. 22, no. 1, pp. 2645, 2013.

[4] M. D. Gallego, P. Luna, and S. Bueno, "User acceptance model of open source software," Computers in Human Behavior, vol. 24, no. 5, pp. 2199-2216, 2008.
[5] V. Midha and P. Palvia, "Factors affecting the success of Open Source Software," Journal of Systems and Software, vol. 85, no. 4, pp. 895-905, 2012.

[6] J. Wang, "Survival factors for Free Open Source Software projects: a multi-stage perspective," European Management Journal, vol. 30, no. 4, pp. 352-371, 2012.

[7] T. van der Valk, M. M. H. Chappin, and G. W. Gijsbers, "Evaluating innovation networks in emerging technologies," Technological Forecasting and Social Change, vol. 78, no. 1, pp. 2539, 2011.

[8] S. L. Toral, M. R. Martínez-Torres, and F. Barrero, "Analysis of virtual communities supporting OSS projects using social network analysis," Information and Software Technology, vol. 52, no. 3, pp. 296-303, 2010.

[9] M. D. König, S. Battiston, M. Napoletano, and F. Schweitzer, "Recombinant knowledge and the evolution of innovation networks," Journal of Economic Behavior \& Organization, vol. 79, no. 3, pp. 145-164, 2011.

[10] J. Ye and A. Kankanhalli, "Exploring innovation through open networks: a review and initial research questions," IIMB Management Review, vol. 25, no. 2, pp. 69-82, 2013.

[11] M. R. Martínez-Torres, "Application of evolutionary computation techniques for the identification of innovators in open 
innovation communities," Expert Systems with Applications, vol. 40, no. 7, pp. 2503-2510, 2013.

[12] H. Wi, S. Oh, and M. Jung, "Virtual organization for open innovation: semantic web based inter-organizational team formation," Expert Systems with Applications, vol. 38, no. 7, pp. 8466-8476, 2011

[13] M. Bianchi, A. Cavaliere, D. Chiaroni, F. Frattini, and V. Chiesa, "Organisational modes for Open Innovation in the biopharmaceutical industry: an exploratory analysis," Technovation, vol. 31, no. 1, pp. 22-33, 2011.

[14] H. Choi, S.-H. Kim, and J. Lee, "Role of network structure and network effects in diffusion of innovations," Industrial Marketing Management, vol. 39, no. 1, pp. 170-177, 2010.

[15] C. Raasch, V. Lee, S. Spaeth, and C. Herstatt, "The rise and fall of interdisciplinary research: the case of open source innovation," Research Policy, vol. 42, no. 5, pp. 1138-1151, 2013.

[16] W. Scacchi, "Issues and experiences in modeling open source software development processes," in Proceedings of the 3rd ICSE Workshop on Open Source Software Engineering, pp. 121-125, May 2003.

[17] Q. Le and J. H. Panchal, "Building smaller sized surrogate models of complex bipartite networks based on degree distributions," IEEE Transactions on Systems, Man, and Cybernetics, Part A: Systems and Humans, vol. 42, no. 5, pp. 1152-1166, 2012.

[18] W. Sack, F. Détienne, N. Ducheneaut, J.-M. Burkhardt, D. Mahendran, and F. Barcellini, "A methodological framework for socio-cognitive analyses of collaborative design of open source software," Computer Supported Cooperative Work, vol. 15, no. 23, pp. 229-250, 2006.

[19] F. Barcellini, F. Détienne, J.-M. Burkhardt, and W. Sack, "A socio-cognitive analysis of online design discussions in an Open Source Software community," Interacting with Computers, vol. 20, no. 1, pp. 141-165, 2008.

[20] C. Haythornthwaite, "Crowds and communities: light and heavyweight models of peer production," in Proceedings of the 42nd Hawaii International Conference on System Sciences, pp. 110, IEEE Computer Society, Los Alamitos, Calif, USA, January 2009, https://www.ideals.uiuc.edu/handle/2142/9457.

[21] Z. H. Li and N. Wang, "Modeling of the dynamical mechanism of peer production based on system dynamics," Studies in Science of Science, vol. 30, no. 2, pp. 232-240, 2012.

[22] J. Yang, C. Yao, W. Ma, and G. Chen, "A study of the spreading scheme for viral marketing based on a complex network model," Physica A: Statistical Mechanics and Its Applications, vol. 389, no. 4, pp. 859-870, 2010.

[23] R. Badawy, B. Hirsch, and S. Albayrak, "Agent-based coordination techniques for matching supply and demand in energy networks," Integrated Computer-Aided Engineering, vol. 17, no. 4, pp. 373-382, 2010.

[24] F. Ponci, L. Cristaldi, M. Faifer, and M. Riva, "Multi agent systems: an example of power system dynamic reconfiguration," Integrated Computer-Aided Engineering, vol. 17, no. 4, pp. 359372,2010 .

[25] J. Li and Z. Sheng, "A multi-agent model for the reasoning of uncertainty information in supply chains," International Journal of Production Research, vol. 49, no. 19, pp. 5737-5753, 2011.

[26] H. Y. K. Lau and S. O. Woo, "An agent-based dynamic routing strategy for automated material handling systems," International Journal of Computer Integrated Manufacturing, vol. 21, no. 3, pp. 269-288, 2008.
[27] N. Ruiz, A. Giret, V. Botti, and V. Feria, "Agent-supported simulation environment for intelligent manufacturing and warehouse management systems," International Journal of Production Research, vol. 49, no. 5, pp. 1469-1482, 2011.

[28] F. C. Yáñez, J.-M. Frayret, F. Léger, and A. Rousseau, "Agentbased simulation and analysis of demand-driven production strategies in the timber industry," International Journal of Production Research, vol. 47, no. 22, pp. 6295-6319, 2009.

[29] J. W. Yin, W. Y. Zhang, and M. Cai, "Weaving an agent-based Semantic Grid for distributed collaborative manufacturing," International Journal of Production Research, vol. 48, no. 7, pp. 2109-2126, 2010.

[30] P. Renna, "Multi-agent based scheduling in manufacturing cells in a dynamic environment," International Journal of Production Research, vol. 49, no. 5, pp. 1285-1301, 2011.

[31] J.-S. Lin, C. Ou-Yang, and Y.-C. Juan, “Towards a standardised framework for a multi-agent system approach for cooperation in an original design manufacturing company," International Journal of Computer Integrated Manufacturing, vol. 22, no. 6, pp. 494-514, 2009.

[32] M. Niazi and A. Hussain, "Agent-based computing from multiagent systems to agent-based models: a visual survey," Scientometrics, vol. 89, no. 2, pp. 479-499, 2011.

[33] R. E. Levitt, "Overview of the Virtual Design Team (VDT): A Computational Model of Project Teams," 2009.

[34] P. Faas, S. Swindler, J. Lyons, R. Levitt, M. Ramsey, and P. Vincent, Organizational Modeling and Simulation in a Planning Organization, Air Force Research Laboratory, Wright-Patterson Air Force Base, Dayton, Ohio, USA, 2009.

[35] J. Martínez-Miranda and J. Pavón, "An agent-based simulation tool to support work teams formation," in International Symposium on Distributed Computing and Artificial Intelligence 2008 (DCAI '08), vol. 50 of Advances in Soft Computing, pp. 80-89, Springer, Berlin, Germany, 2009.

[36] R. Garcia, "Uses of agent-based modeling in innovation/new product development research," Journal of Product Innovation Management, vol. 22, no. 5, pp. 380-398, 2005.

[37] J. X. Wang, M. X. Tang, L. N. Song, and S. Q. Jiang, “Design and implementation of an agent-based collaborative product design system," Computers in Industry, vol. 60, no. 7, pp. 520-535, 2009.

[38] J. H. Panchal, "Agent-based modeling of mass-collaborative product development processes," Journal of Computing and Information Science in Engineering, vol. 9, no. 3, pp. 1-12, 2009.

[39] Y.-Z. Li, S. Zhang, X.-D. Zhang, and T. Wang, "Agent model for mass collaborative product development process and its simulation application," Computer Integrated Manufacturing Systems, vol. 19, no. 8, pp. 1948-1956, 2013.

[40] R. Rudek, "Computational complexity and solution algorithms for flowshop scheduling problems with the learning effect," Computers \& Industrial Engineering, vol. 61, no. 1, pp. 20-31, 2011. 


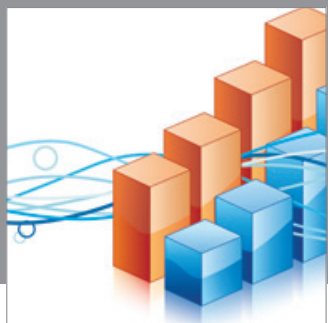

Advances in

Operations Research

mansans

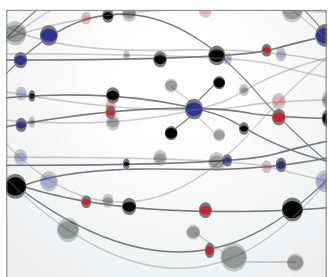

The Scientific World Journal
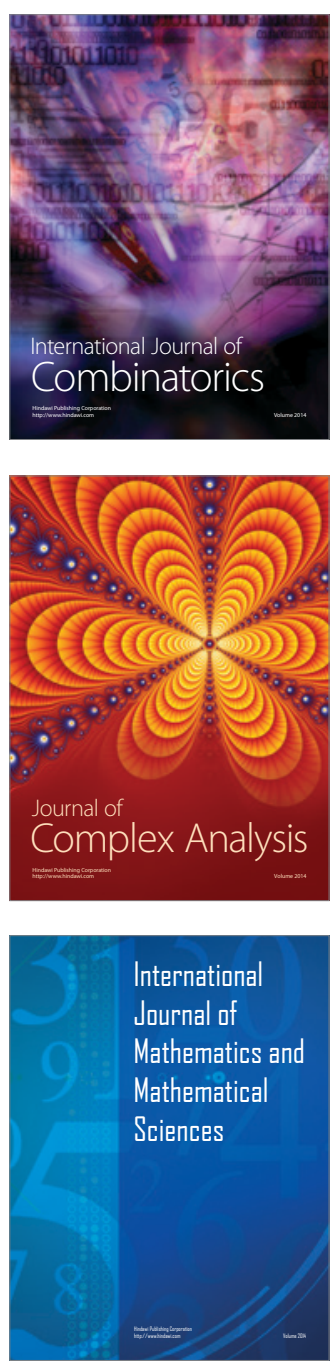
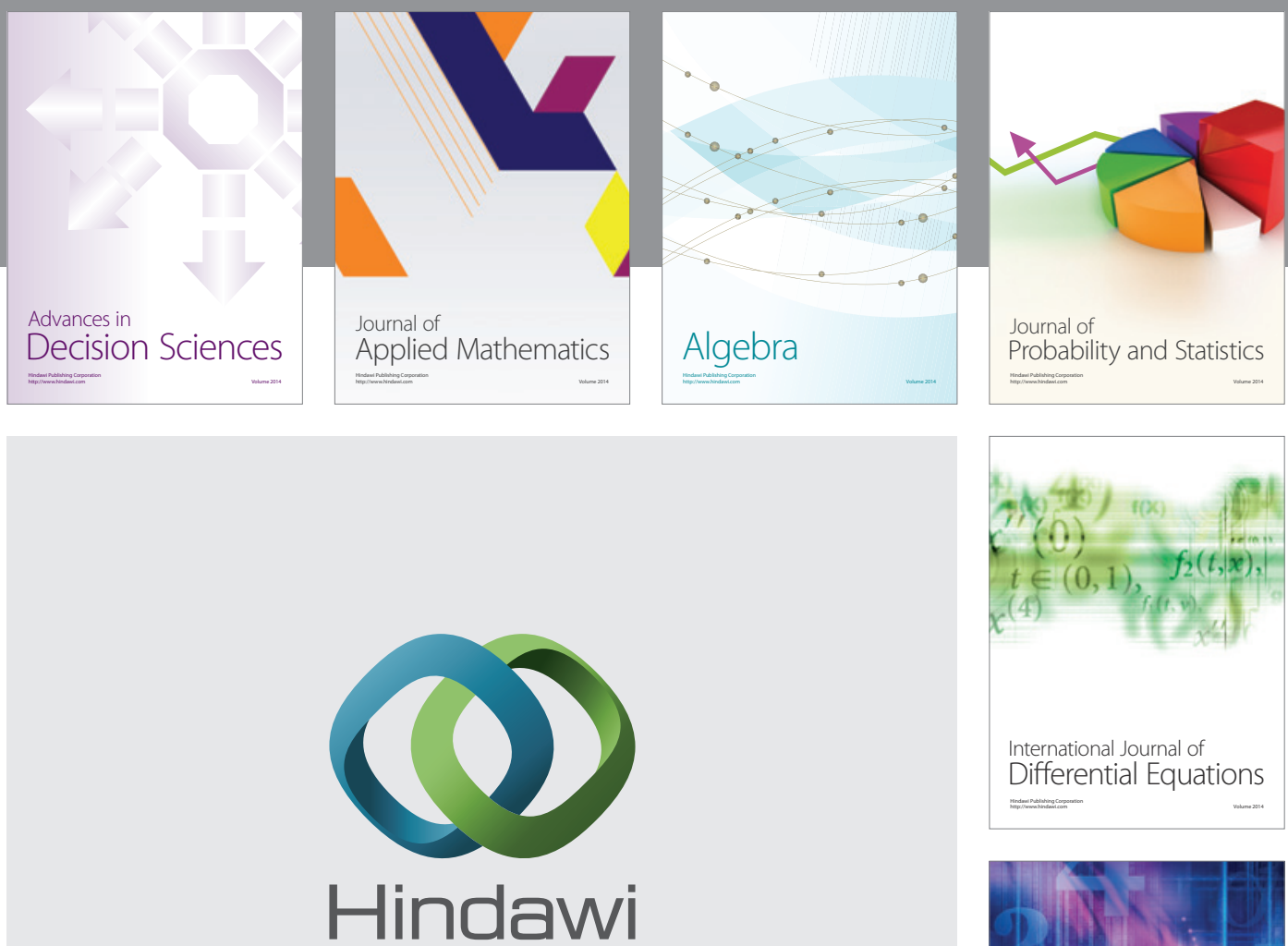

Submit your manuscripts at http://www.hindawi.com


Journal of

Function Spaces

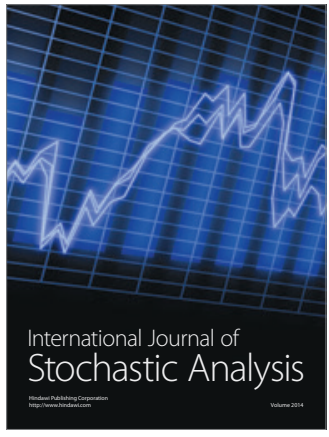

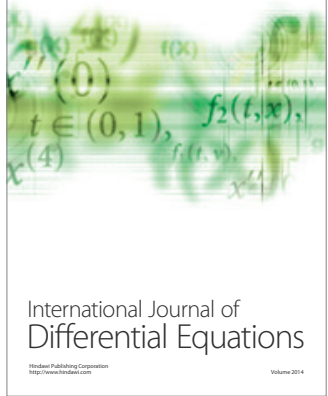
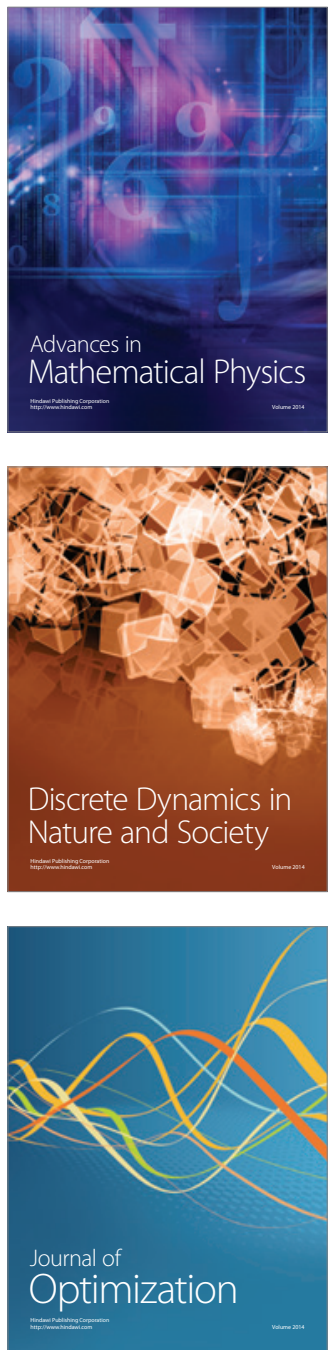